\title{
Evidence-based decision-making for primary care: The interpretation and role of pragmatic trials
}

In the era of evidence-based medicine, health authorities are obligated to base policies on solid evidence. The gold standard of evidence-based medicine is the randomised controlled trial (RCT) in which participants fulfil strict criteria for inclusion, and the ecology of care - i.e. factors other than the intervention that could influence the outcome are controlled. ${ }^{[1]}$ However, evidence supporting systems interventions in health services that involve multiple changes in the delivery of services is complex and requires the synthesis of evidence from multiple sources. Not surprisingly, such evidence is scarcest in the resource-constrained settings that need it most, where issues of prioritisation and cost-effectiveness are of paramount importance.

This dilemma facing health authorities is well illustrated by circumstances surrounding the adoption of Adult Primary Care (APC) by the National Department of Health for use in primary care facilities in South Africa (SA), which forms part of the Ideal Clinic initiative. ${ }^{[2]}$ APC is the new name for Primary Care 101 (PC101), developed by the Knowledge Translation Unit at the University of Cape Town Lung Institute, after more than a decade of formative research. APC is a comprehensive clinical management guide and training approach for frontline clinicians (in SA, usually nurses), providing simple algorithms for the integrated management of multiple conditions; in fact the majority of reasons for which adult patients attend primary care facilities, including preventive, curative and chronic care. ${ }^{[3]}$ What evidence might the health authorities have considered in adopting the APC approach, particularly in the face of the recent publication, in a high-impact journal, of a large pragmatic randomised trial conducted in 38 clinics in the Eden and Overberg districts of SA, which failed to show significant improvement in the management of three non-communicable diseases (NCDs) and case detection of depression following the introduction of PC101? ${ }^{[4]}$ What other factors might the health authorities have considered in making this decision?

First, it should be noted that the decision predated the availability of the results of the PC101 trial. We argue that there are at least three reasons why the decision remains appropriate: (i) methodological issues in pragmatic controlled trials such as the PC101 trial; (ii) additional evidence supporting the effectiveness of PC101; and (iii) the role of evidence in shaping health system reform.

In the continuum of research methods from observational studies to randomised trials, the pragmatic trial comes close to 'reallife' conditions while retaining the advantages of randomisation and preservation of internal validity. Explanatory RCTs, where interventions are tested under tightly controlled conditions, are useful to decision-makers when they produce negative results, as interventions that do not work under ideal conditions are unlikely to be effective when applied in real-world settings, and so can be abandoned. When they produce positive findings, questions arise as to whether they will yield similar results under routine circumstances where, for example, adherence is usually far lower than what was achieved in the trial. Conversely, positive pragmatic trials provide compelling evidence of usefulness and should prompt adoption. When negative, further research remains an option, particularly if, as in the PC101 trial, there is no evidence of harm. ${ }^{[5]}$

The PC101 trial was ambitious in its pragmatic orientation and affected by the unpredictable impact of real-life conditions. It focused on the intensification of prescribing for three NCDs - hypertension, diabetes and chronic respiratory diseases - and case detection of depression, while adopting a 'hands-off' approach to assessing the validity of correct diagnosis, eligibility for treatment intensification, underlying disease severity, or patient adherence to treatment. Furthermore, factors external to the trial played a role. For example, during the trial, the district health authorities, in part inspired by a renewed focus on NCDs that accompanied PC101, implemented a Chronic Disease Season to encourage recognition and treatment of NCDs in all clinics, including 17 control clinics in the Eden district.

These and other issues described in the main publication probably accounted for the negative results for the primary endpoints. However, notably, analysis of prespecified subgroups - patients with poor control of diabetes and those with highest blood pressures benefited significantly from the intervention. ${ }^{[4]}$

The challenges of designing and performing pragmatic controlled trials of complex interventions such as PC101, together with the scarcity or small size of positive outcomes, explain their rarity, particularly in low-income settings. But the PC101 trial is the latest of a succession of research reported by the Knowledge Translation Unit in more than 30 publications over 12 years. This research includes three other pragmatic randomised trials that showed consistent, reproducible improvements in both process and health outcomes, particularly for infectious diseases. ${ }^{[6-8]}$ The PC101 trial must therefore be seen in the context of a growing experience of integrated nurseled primary care, in what has recently been described as a Learning Health System approach. ${ }^{[9]}$ The Learning Health System is one that aims 'to integrate delivery of health services with the generation of new knowledge about the effectiveness of these services'. Since the first version of PC101 (initially called the Practical Approach to Lung Health in South Africa - PALSA), results from studies of varying design - observational, qualitative, cost-effectiveness, and pragmatic randomised controlled trials - have been used to improve and expand the clinical guide and training methods. Further trials are underway in North West Province, SA (testing a strengthened mental health component of APC) and in Brazil (testing a Brazilian adaptation). The utility and role of APC should therefore not be invalidated by the results of one negative trial.

Finally, how might health authorities weigh evidence from various sources in their important policy decisions? An evidence hierarchy should place pragmatic trials performed in the target health facilities and embedded in usual practice as the highest level of evidence. Sadly, because pragmatic trials are complex and their results often challenging to interpret, decision-makers rely on findings from less robust research designs such as before-and-after observational studies. Supportive evidence from research by an independent party provides further assurance for decision-makers. PC101 was subjected to a controlled before-and-after study with a limited survey of impact on nurse knowledge and quality of care in 42 clinics in three health districts of SA. Findings were broadly positive across a range of process outcomes. Furthermore, since quality care can only be delivered by trained and motivated staff, qualitative research and audit confirming the rapid and enthusiastic uptake and rollout among healthcare workers of the APC approach, including most recently among undergraduate doctors, also serve as validation of the approach. 
What other factors require consideration? Cost and competing priorities are major factors in decision-making. Although a formal cost-consequence analysis of PC101 has not been completed, previous research results point to little if any additional cost, other than that relating to treatment related to improved detection of infectious disease. ${ }^{[10]}$

The attractiveness of the approach in implementing APC is that it utilises existing staff and staff trainers, and simply involves standardisation of clinical management and healthcare worker training. The training programme employs adult learning principles that increase confidence and strengthen self-perceived efficacy of healthcare workers. Furthermore, regular updates of the clinical management guide avoid the confusion of outdated guidelines and policies. The lack of alternatives to address the heavy load and multimorbidity faced by frontline clinicians, reflecting the collision of SA's infectious disease and NCD epidemics, supports the adoption and implementation of APC. More than $80 \%$ of this load is borne by nurses in primary care in SA, a burden for which they often feel ill equipped. APC is designed around their needs and scope of practice, customised for their use in the clinic and compliant with all policies, and, importantly, harmonises conflicting instructions in different official guidelines.

Health systems research is a challenging but vital component of healthcare delivery, especially in resource-constrained settings, in which bad choices are both wasteful and deny care to those who need it. Results of a single well-conducted pragmatic controlled trial are of unquestionable value, but the evidence gap is best addressed by a Learning Health System - a continuous programme of research designed to generate and apply best evidence and then rigorously evaluate its effectiveness. Such a programme requires long-term collaborations between researchers, healthcare system and authorities to drive the process of discovery as a natural outgrowth of patients' care; and to ensure innovation, quality, safety and value in health care.[11]

\section{Lara R Fairall}

Knowledge Translation Unit, University of Cape Town Lung Institute, and Department of Medicine, Faculty of Health Sciences, University of Cape Town, South Africa lara.fairall@uct.ac.za

\section{Ozayr Mahomed}

Discipline of Public Health Medicine, School of Clinical Medicine, College of Health Sciences, Nelson R Mandela School of Medicine, University of KwaZulu-Natal, Durban, South Africa

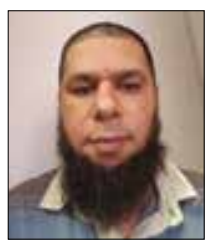

\section{Eric D Bateman}

Knowledge Translation Unit, University of Cape Town Lung Institute, South Africa

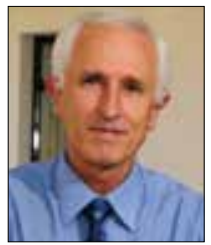

1. Price D, Bateman ED, Chisholm A, et al. Complementing the randomized controlled trial evidence base: Evolution not revolution. Ann Am Thorac Soc 2014;11(Suppl):S92-S98. https://doi.org/10.1513/ AnnalsATS.201308-276RM

2. Ideal Clinic South Africa. Ideal Clinic Manual Version 16. Pretoria: National Department of Health, South Africa, October 2015

3. Mash B, Fairall LR, Adejayan O, et al. A morbidity survey of South African primary care. PLoS One 2012;7(3):e32358. https://doi.org/10.1371/journal.pone.0032358

4. Fairall LR, Folb N, Timmerman V, et al. Educational outreach with an integrated clinical tool for nurseled non-communicable chronic disease management in primary care in South Africa: A pragmatic cluster randomized controlled trial. PLoS Med 2016;13(11):e1002178. https://doi.org/10:1371/journal. pmed. 1002178

5. Sackett DL. Clinician-trialist rounds: Mind your explanatory and pragmatic attitudes! Part 1: What. Clin Trials 2013;10(3):495-498. https://doi.org/10.1177/1740774513484395

6. Fairall LR, Zwarenstein M, Bateman ED, et al. Effect of educational outreach to nurses on tuberculosis case detection and primary care of respiratory illness: Pragmatic cluster randomised controlled trial. BMJ 2005;331:750. https://doi.org/10.w1136/bmj.331.7519.750

7. Zwarenstein M, Fairall LR, Lombard C, et al. Outreach education for integration of HIV/AIDS care, antiretroviral treatment, and tuberculosis care in primary care clinics in South Africa: PALSA PLUS pragmatic cluster randomised trial. BMJ 2011;342:d2022. https://doi.org/10.1136/bmj.d2022

8. Fairall LR, Bachmann MO, Lombard C, et al. Task shifting of antiretroviral treatment from doctors to primary-care nurses in South Africa (STRETCH): A pragmatic, parallel, cluster-randomised trial.
to Lancet 2012;380(9845):889-898. https://doi.org/10.1016/S0140-6736(12)60730-2

9. English M, Irimu G, Agweyu A, et al. Building Learning Health Systems to accelerate research and improve outcomes of clinical care in low- and middle-income countries. PLoS Med 2016;13(4):e1001991. https://doi.org/10.1371/journal.pmed.1001991

10. Fairall L, Bachmann MO, Zwarenstein M, et al. Cost-effectiveness of educational outreach to primary care nurses to increase tuberculosis case detection and improve respiratory care: Economic evaluation alongside a randomised trial. Trop Med Int Health 2010;15(3):277-286. https://doi.org/10.1111/j.13653156.2009.02455.x

11. Olsen L, Aisner D, McGinnis JM. The Learning Healthcare System: Workshop Summary (IOM Roundtable on Evidence-Based Medicine). Washington, DC: National Academies Press, 2007. 